\title{
Lightweight Potential of 3D Endless Fiber Reinforcement of Polyurethane Foam Cores with Spacer Fabrics in Hybrid Sandwich Structures with Fiber Reinforced Thermoplastic Facings
}

\author{
Kay Schäfer ${ }^{1, a^{*}}$, Daisy Nestler ${ }^{1, b}, K^{2}$ ristina Jahn ${ }^{1, c}$, \\ Henrik Jentzsch ${ }^{1, \mathrm{~d}}$ and Lothar Kroll ${ }^{2, \mathrm{e}}$
${ }^{1}$ Endowed Chair Textile Plastic Composites and Hybrid Compounds, Faculty of Mechanical Engineering, Technical University of Chemnitz, D-09126 Chemnitz, Germany
${ }^{2}$ Department of Lightweight Structures and Polymer Technology, Faculty of Mechanical Engineering, Technical University of Chemnitz, D-09126 Chemnitz, Germany
akay.schaefer@mb.tu-chemnitz.de, bdaisy.nestler@mb.tu-chemnitz.de, ckristina.jahn@mb.tu-chemnitz.de, dhenrik.jentzsch.extern@veritas-ag.de, elothar.kroll@mb.tu-chemnitz.de

\begin{abstract}
Keywords: sandwich design, polyurethane foam, spacer fabric, thermoplastic composite, reaction injection moulding

Abstract. Sandwich structures consisting of fibre-reinforced plastic (FRP) facings and core are ideally suited as substitution materials for reducing component masses. The endless fibre reinforcement has the greatest performance potential. Both thermoset and thermoplastics are already being processed into endless fibre-reinforced sandwich facings according to the state of the art. The 3D endless fibre reinforcement of cores is a current research topic. This paper describes the development of a hybrid sandwich consisting of thermoplastic composite facings and an innovative core composite. This is made of polyurethane (PUR) rigid or flexible foam, which is reinforced with spacer fabric. The sandwich manufacturing in Reaction Injection Moulding (RIM) includes the original forming of the core and the simultaneous bonding of the facings. This efficient process offers the potential for the production of such complex structures in medium or large series. The sandwich structures and their individual components were characterised in the standardised compression and bending test. The lightweight potential of spacer fabric reinforcement is demonstrated by comparing the specific mechanical properties of sandwich structures with and without core reinforcement. In comparison to reinforced and unreinforced foams, the effect of sandwich design is also shown.
\end{abstract}

\section{Introduction and State of the Art}

The lightweight design reduces the mass of components while maintaining product functionality. This leads to reduced $\mathrm{CO}_{2}$ emissions in the case of moving masses. In the form lightweight design the material distribution in components is optimised [1]. This is used by the sandwich design. The determination of materials with the lowest possible density for the requirements of a component is carried out in the material lightweight design [1]. FRP are about $40 \%$ lighter than aluminium and up to $80 \%$ lighter than steel [2]. The optimisation of the fibre orientation with regard to the mechanical requirements leads to large weight savings. Endless fibre reinforcement has the greatest performance potential. A wide range of fibres and plastics are available on the market. Different requirement profiles can be fulfilled by a targeted selection. Sandwich structures consisting of endless fibrereinforced plastic facings and cores are therefore ideally qualified as substitution material for reducing component masses. Only foams are suitable for this composition as core type. Those alone can transfer forces between the fibres. At the same time they have a low density because of their porosity in accordance with the requirements of a sandwich core.

Completely thermoset FRP sandwich structures offer higher mechanical properties and higher temperature stability compared to completely thermoplastic sandwich structures [3]. This are in contrast easily recyclable [3]. Components made of thermoplastic facings and a thermoset core can 
be designed very freely [4]. A good forming ability of the thermoplastics and the high flowability of thermoset foams are the reasons for this. In addition with foam injection moulding no shrinkage or warpage of moulded parts occurs even by very complex forms [5]. This paper describes scientific investigations of this combination.

Thermoplastics are already being processed into endless fibre-reinforced sandwich facings according to the state of the art [6]. The 3D endless fibre reinforcement of foam cores is a current research topic in contrast. Only short [7] and long fibres [8] can be processed in Reinforced Reaction Injection Moulding (RRIM) for the production of thermosets. In Structural Reaction Injection Moulding (SRIM) only 2D textiles have been processed up to now [9].

Spacer fabrics are 3D fibre structures consisting of two fabric surfaces which are kept at a defined distance by spacer threads [10]. The suitability in principle of these structures for the reinforcement of PUR foams has already been investigated [11-13]. This paper deals with the addition of such core composites with endless fibre-reinforced thermoplastic facings to sandwiches. The development of efficient processes for the production of such complex structures for medium and large series is challenging [14]. The number of process steps has to be minimised. Hybrid sandwich structures meet this requirement. They are manufactured by bonding the faces with the core simultaneously with the original forming of a sandwich layer [15]. This was realised in that investigation. Core composites with spacer fabric reinforcement were manufactured in the in-situ foaming with polyurethane and simultaneously bonded to thermoplastic facings. This is based on own preliminary work. On the one hand these are studies on the adhesive strength between PUR foams and thermoplastics which were bonded in the RIM [16]. This enables the selection of suitable materials for loadable adhesive bondings in the sandwich. On the other hand the textile surface design of thermoplastic FRP was used [17]. This provides a highly loadable transition area between the facings and the core. Focus of this paper are the specific mechanical properties that sandwich structures with spacer fabric reinforcement and their individual components achieve. The lightweight potential of the 3D endless fibre reinforcement was quantified by comparing the values of unreinforced and reinforced cores and sandwiches.

\section{Experimental Part}

Sandwich design and materials. The investigations of this paper were carried out on sandwich composites according to Fig. 1, a) consisting of thermoplastic composite facings and an innovative core composite. This is made of polyurethane foam, which is reinforced with a spacer fabric. The focus was on a sandwich thickness of $40 \mathrm{~mm}$ in order to demonstrate the potential of spacer fabrics for $3 \mathrm{D}$ endless fibre reinforcement of thick cores for special thermal and sound insulation requirements.

a)

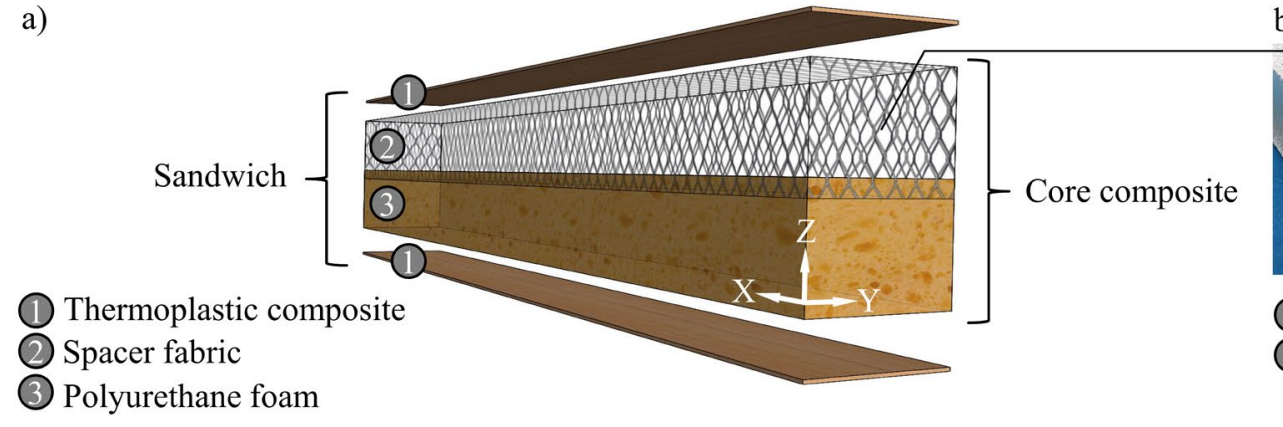

b)

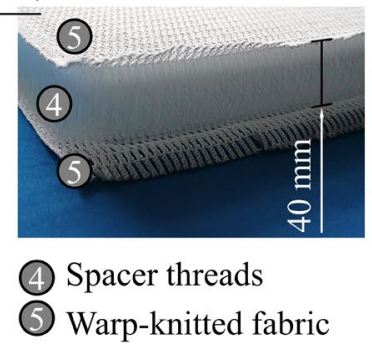

Fig. 1: a) Exploded view of the investigated sandwich composite and b) spacer fabric

The thermoplastic composite was manufactured by SKL Schwergewebekonfektion Lichtenstein $\mathrm{GmbH}$ (Oberlungwitz, Germany) using the OLU-Preg ${ }^{\circledR}$ process [18]. It is about $1.5 \mathrm{~mm}$ thick, has a density of approximately $1500 \mathrm{~kg} / \mathrm{m}^{3}$ and a theoretical fibre volume content of $42 \%$. As reinforcement structure it contains non-crimp fabrics of glass which have a roving fineness of 
300 tex, a filament diameter of $14 \mu \mathrm{m}$ and a grammage of $200 \mathrm{~g} / \mathrm{m}^{2}$. Four of them were processed in $\mathrm{X}$ and Y direction each. The matrix is formed by PP. On one side of the textile layer stack for the production of the thermoplastic composite no final layer of matrix was used. This results in partially unimpregnated glass fibres on this side, which are to a sufficient extent embedded in the plastic matrix to be mechanically stressed. This microporous surface structuring offers best conditions for the bonding of the sandwich core.

A sandwich core thickness of $40 \mathrm{~mm}$ can only be reinforced 3D endless with spacer fabrics produced on HighDistance ${ }^{\circledR}$ double raschel machines. A corresponding variant from Spiga GmbH (Flöha, Germany) was selected for this study (Fig. 1, b). It consists of polyester yarns. The surfaces are made of multifilaments as fillets with a tricot lapping. The surface porosity is $8 \%$ and was determined by microscopy. The slight porosity of the threads was neglected. The spacer threads consist of monofilaments with a diameter of $0.2 \mathrm{~mm}$. In the 3D fibre structure they run IXI. The low weight per volume of the spacer fabric of $62 \mathrm{~kg} / \mathrm{m}^{3}$ and the low fibre volume content of $4.4 \%$ with a spacer thread number of 520/in ${ }^{2}$ justify its potential as a light but high-performance reinforcing structure in the core of FRP sandwich structures.

Two reactive 2-component cold foam systems from Covestro AG (Leverkusen, Germany) were used to manufacture the PUR foams (Table 1). They produce a flexible and a rigid PUR foam which each have a free foam weight per volume of approximately $40 \mathrm{~kg} / \mathrm{m}^{3}$. The flexible foam system primarily produces open and the rigid closed cells. Both systems consist of an aromatic polyisocyanate and a polyol formulation, which contains polyether polyol and all necessary additives for the polyaddition reaction and $\mathrm{CO}_{2}$ formation. The flexible foam system with a starting time of $8 \mathrm{~s}$ and a tack-free time of 4 minutes has the considerably faster process times compared to the rigid foam system with a starting time of $50 \mathrm{~s}$ and a tack-free time of 15 minutes.

Table 1: Applied PUR foam systems

\begin{tabular}{r|l|r|r}
\multicolumn{2}{l|}{} & Polyurethane rigid foam & Polyurethane flexible foam \\
\hline \multirow{2}{2}{} & Name & BAYDUR 43-101 & PU Blend RP.PU 90WF99 \\
\cline { 2 - 4 } & OH number [mg KOH/g] & 330 & $39 \pm 3$ \\
\cline { 2 - 4 } & Weight ratio [\%/wt.] & 67 & 100 \\
\hline \multirow{2}{*}{} & Name & Desmodur 44V20 LF & Desmodur VP.PU 3230 \\
\cline { 2 - 4 } & NCO content [\%/wt.] & $30.5-32.5$ & $32.0-33.0$ \\
\cline { 2 - 4 } & Weight ratio [\%/wt.] & 100 & 54 \\
0 & & &
\end{tabular}

Manufacturing process. An aluminium tool with a cavity of $500 \mathrm{~mm}$ length, $50 \mathrm{~mm}$ width and $40 \mathrm{~mm}$ height was used to manufacture samples. It has a 4-unit runner sprue that feeds the cavity over its complete length at a height of $15 \mathrm{~mm}$. It was heated to $42{ }^{\circ} \mathrm{C}$ for the sample manufacturing. MP-4066 from Münch Chemie International GmbH (Weinheim, Germany) was used as release agent.

The thermoplastic composites were cut with a water jet system and the spacer fabrics with the USM-G electric circular blade from Hoogs Cutting Systems GmbH \& Co. KG (Bonn, Germany) into the cavity area of $500 \mathrm{~mm} \times 50 \mathrm{~mm}$. The longer side (X direction in Fig. 1, a) of the spacer fabric corresponded to the working direction in the warp knitting process.

The two-component low pressure system M8/2 from Unipre GmbH (Werl, Germany) was used for mixing and injection of the foam systems. An output of $700 \mathrm{~g} / \mathrm{min}$ was used for the flexible and $600 \mathrm{~g} / \mathrm{min}$ for the rigid foam system. Shortly before the mould is closed and the PUR is filled in, all inserts are placed in the cavity to produce the composite structures. They have room temperature of approximately $23{ }^{\circ} \mathrm{C}$ when the PUR foam flows against them. If no spacer fabrics or thermoplastic composites are used, reference samples without core reinforcement or facings can be produced for the scientific investigations. No fixing of the inserts in the cavity was necessary to prevent displacement because of the low-pressure technology used. The flow of the PUR foam to a complete cavity filling was exclusively caused by the chemical reaction and the resulting blowing agent $\mathrm{CO}_{2}$. The tack-free time of the foam systems defines the time until the samples can be removed from the mould. 
A minimum of $60 \mathrm{~g}$ reaction mass is required for both foam systems to completely fill the cavity with PUR if no inserts are used. The resulting PUR foam beams have a weight per volume of $58 \mathrm{~kg} / \mathrm{m}^{3}$ as a foamed runner sprue weights about $2 \mathrm{~g}$ and the total volume of the cavity is $1000 \mathrm{~cm}^{3}$. The weight per volume of the foams can be specifically adjusted to the system accuracy of $\pm 2.5 \mathrm{~g}$ by introducing increased PUR reaction masses into the mould. If spacer fabrics are used as inserts in the cavity, increased PUR reaction masses have to be used compared to filling the free space. They interfere the PUR foam expansion. Filling the spacer fabric described above with minimal PUR reaction mass results in a foam weight per volume of $88 \mathrm{~kg} / \mathrm{m}^{3}$. This was also specifically used for the production of reference samples without spacer fabric reinforcement for a better comparability. The core composites without facings have a weight per volume of about $150 \mathrm{~kg} / \mathrm{m}^{3}$, the sandwich samples without core reinforcement $203 \mathrm{~kg} / \mathrm{m}^{3}$ and the sandwich samples with core reinforcement $265 \mathrm{~kg} / \mathrm{m}^{3}$.

Testing. The tensile test according to DIN EN ISO 527-4 and the 3-point bending test according to DIN EN ISO 14125 were used to characterise the thermoplastic composite in accordance with the usual market practice.

Two standards were used to determine the compressive properties of the sandwiches and their individual components. The test samples with flexible foam core were tested according to DIN EN ISO 3386-1. This takes the flexibility of materials into account. Three load cycles up to $30 \%$ remaining height are performed before the measurement cycle is recorded. The samples with rigid foam core were tested according to DIN EN ISO 844. This does not include load cycles before the measurement cycle because of the destructibility of material. The two standards do not differ in their main features apart from that. The manufactured beams were cut to the sample dimensions of $50 \mathrm{~mm} \times 50 \mathrm{~mm} \times 40 \mathrm{~mm}$ in accordance with the standards for this material test. 25 measurements per series were carried out.

The 3-point bending test of the sandwiches and their individual components was carried out following the DIN EN ISO 178 on 10 samples per series, which corresponded to the dimensions of the tool cavity. The length did not meet the requirements of the standard. The bending distance was set to $51 \mathrm{~mm}$ and the loading and unloading behaviour was recorded.

\section{Results and Discussion}

Tensile and bending properties of the thermoplastic composite. Table 2 shows the tensile and bending properties of the thermoplastic composite.

Table 2: Mechanical properties of the applied thermoplastic composite

\begin{tabular}{l|r|r|r}
$\mathrm{n}=14$ & Tensile properties & $\begin{array}{l}\text { Bending properties } \\
\text { in X direction }\end{array}$ & $\begin{array}{l}\text { Bending properties } \\
\text { in Y direction }\end{array}$ \\
\hline DIN-Norm & DIN EN ISO 527-4 & DIN EN ISO 14125 & DIN EN ISO 14125 \\
\hline Young's modulus [GPa] & 17.8 & 20.5 & 10.6 \\
\hline SD (Young's modulus) [GPa] & 0.4 & 0.4 & 0.9 \\
\hline Strength [MPa] & 351 & 425 & 319 \\
\hline SD (Strength) [MPa] & 17 & 26 & 15 \\
\hline Breaking elongation [\%] & 2.3 & 2.2 & 3.9 \\
\hline SD (Breaking elongation) [\%] & 0.1 & 0.2 & 0.4
\end{tabular}

SD: standard deviation

Compression properties of the sandwiches and their individual components. The reinforcement of PUR foams with a spacer fabric results in a highly anisotropic deformation behaviour under compressive load corresponding to the curvature of the spacer threads (Fig. 2). 
a)

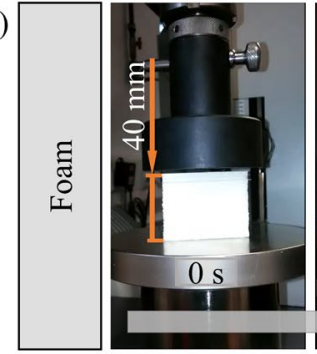

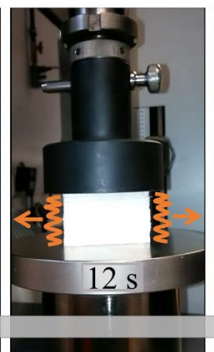

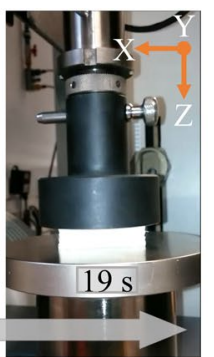

b)
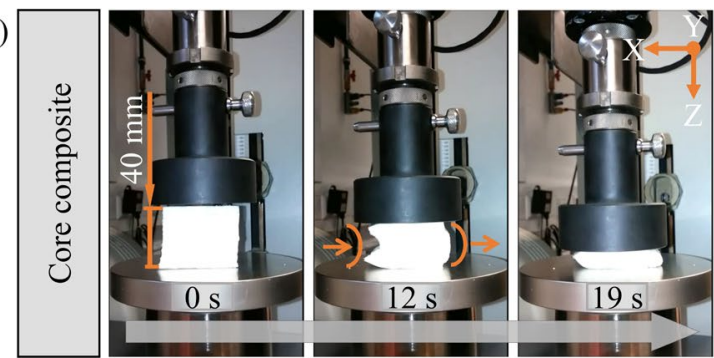

Fig. 2: Deformation behaviour of a) an unreinforced compared to b) a spacer fabric reinforced foam

The spacer fabric as individual component has three powers of ten lower compressive stress values compared to the rigid materials (Fig. 3, a). As reinforcing structure in the core composite it causes a significant increase of the values compared to the reference rigid foam. Adding facings to the core composite leads to further increases in compressive stress. The transverse contraction of the foam is hindered by the connection to the facings, resulting in higher compaction and greater states of stress in the material. The core reinforcement in the sandwich only results at higher degrees of deformation in performance increases. In the mass-related, specific comparison (Fig. 3, b), the sandwich with core reinforcement drops behind the other rigid materials because of the mass proportions of the core reinforcement and the thermoplastic composite facings. The rigid foam and the core composite are equally positioned in the specific comparison and achieve the highest characteristic values. An increase in the lightweight potential is therefore not achieved by reinforcing rigid foam with spacer fabrics when compressive stress is applied.
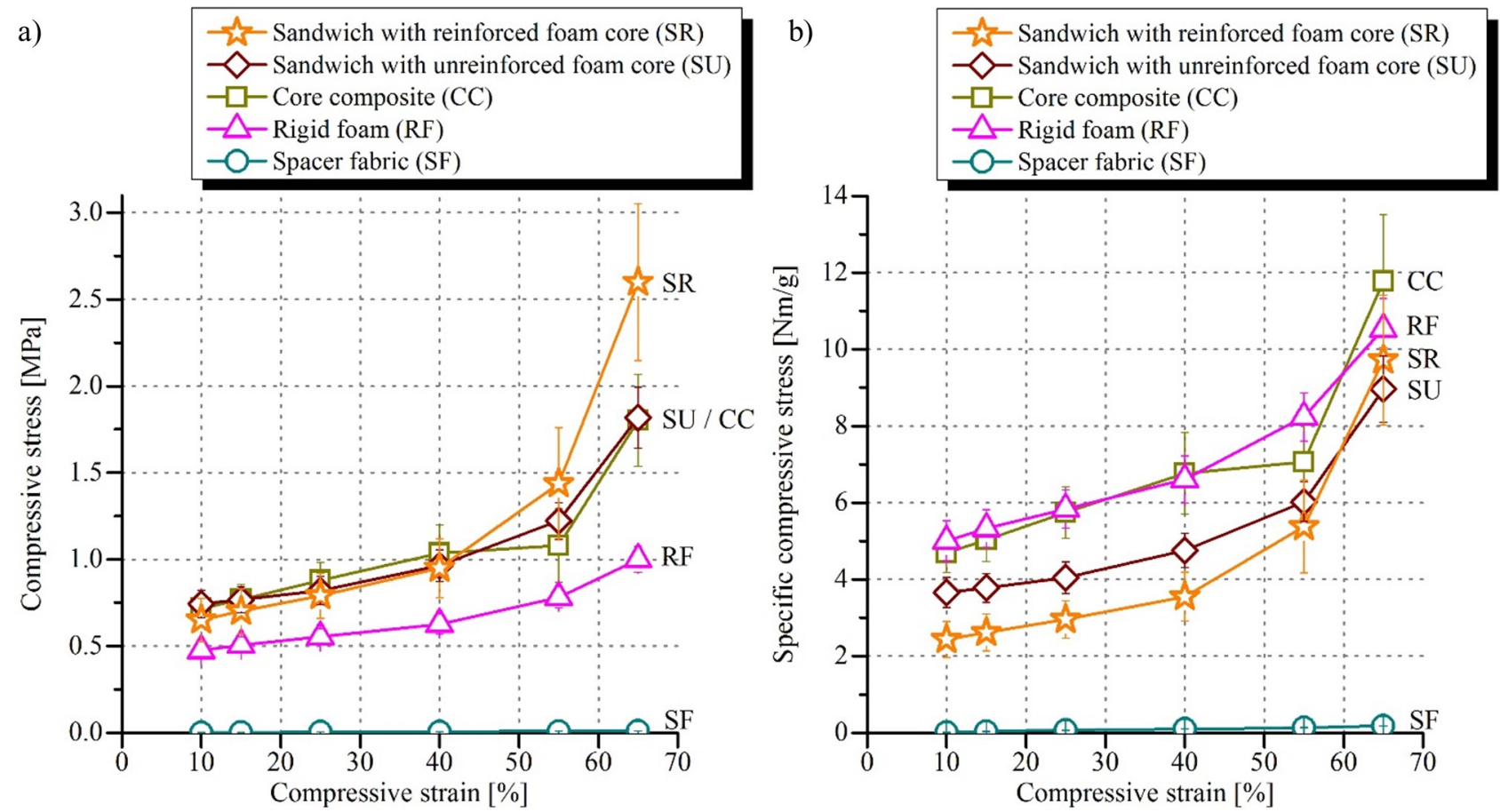

Fig. 3: a) Absolu te and b) specific characteristic values of the pressure test according to DIN EN ISO 844 of the rigid sandwiches their individual components

The spacer fabric also has the lowest compressive stress values compared to the flexible materials investigated (Fig. 4, a). But it leads to a great reinforcing effect in the flexible foam as well as in the sandwich. This is also demonstrated in the specific characteristic value comparison (Fig. 4, b). The lightweight potential of flexible foam is accordingly significantly increased by the reinforcement with spacer fabrics when compressive stress is applied. 

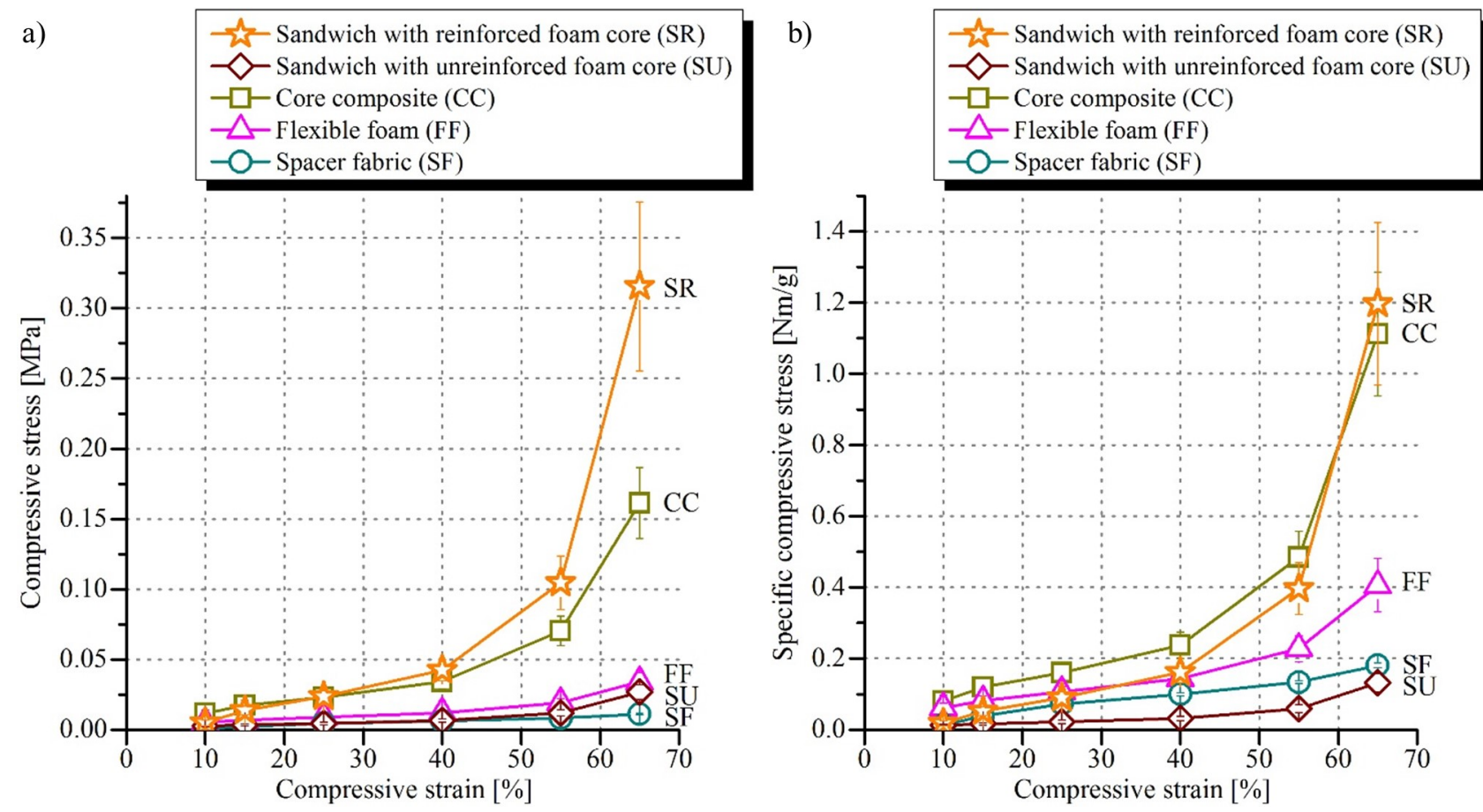

Fig. 4: a) Absolute and b) specific characteristic values of the pressure test according to DIN EN ISO 3386-1 of the flexible sandwiches their individual components

Bending properties of the sandwiches and their individual components. In the bending test of the rigid sandwich without core reinforcement, the thermoplastic composite fails first interlaminar at the point of load application (Fig. 5). The interface between the facings and the core and then the core itself fail as a result of further deformation. The integration of the spacer fabric into the sandwich prevents core failure. The flexible sandwiches do not fail in the bending test. A greater thickness deformation at the point of load application compared to the rest of the sample deformation can be observed without core reinforcement. This is homogenised by the integration of the spacer fabric.
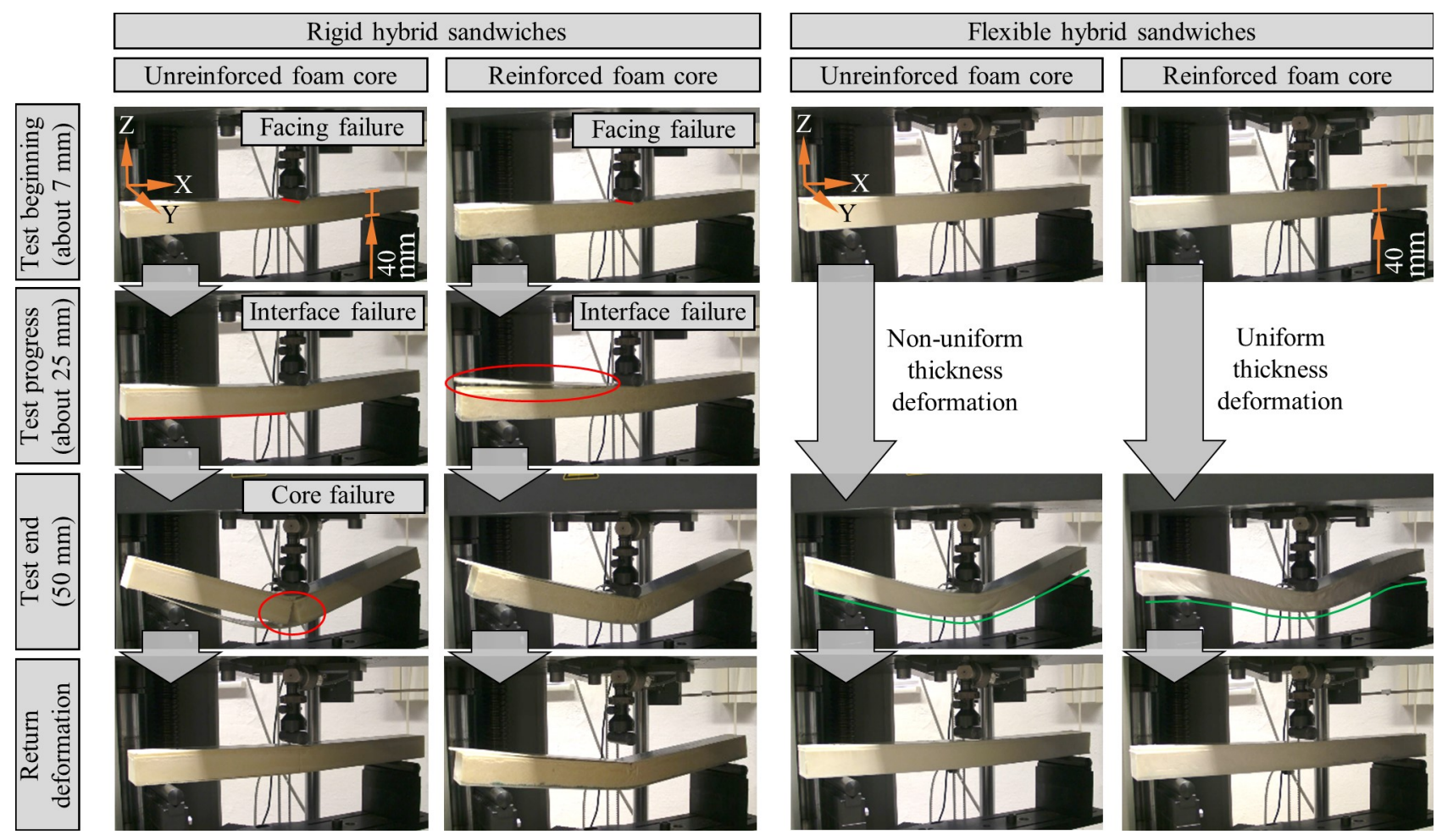

Fig. 5: Deformation behaviour of the hybrid sandwich beams under bending load 
Increasing bending strength and stiffness can be determined for both rigid and flexible materials with increasing material complexity (Fig. 6). The integration of the spacer fabric in the flexible sandwiches also increases the area of the hysteresis curve, which corresponds to the energy absorption of the material. The flexible core composite shows a buckling at approximately $2.2 \%$ bending strain. This is prevented by the addition of facings, as the load is distributed more evenly.
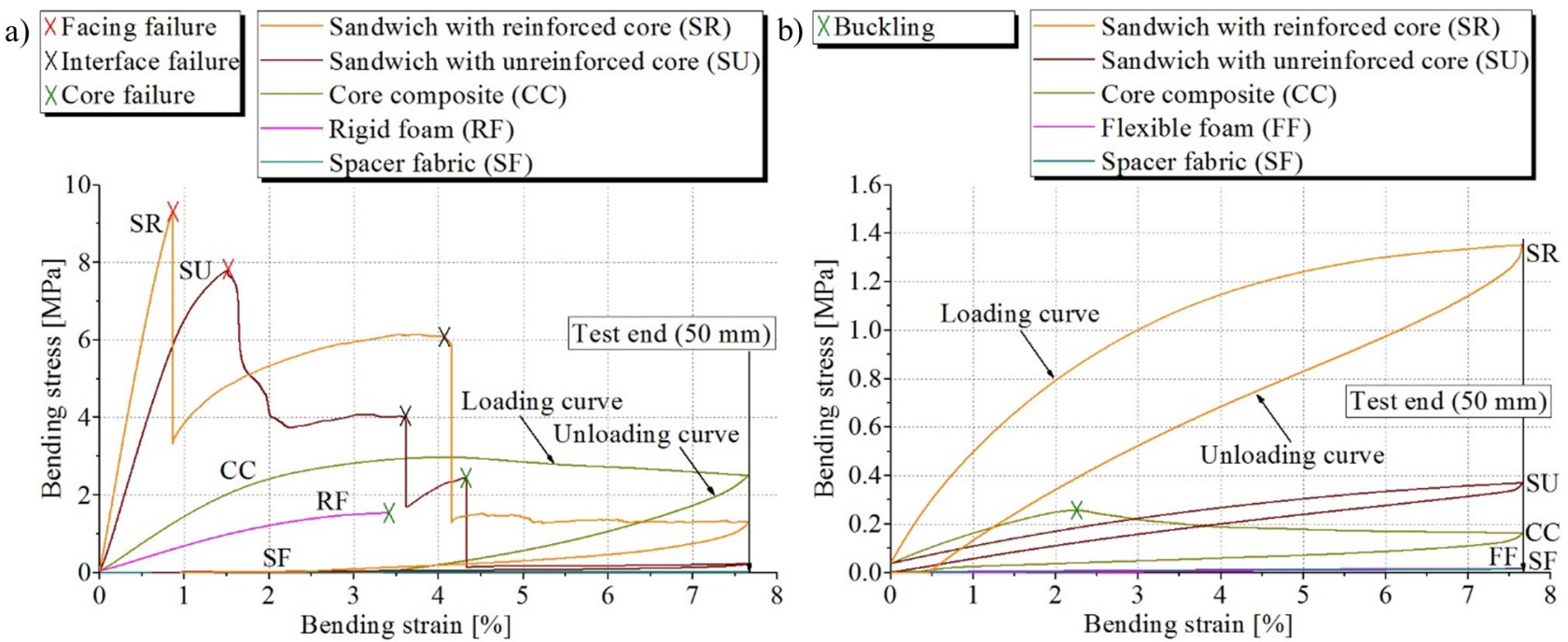

Fig. 6: Exemplary measurement curves of the bending test based on DIN EN ISO 178 of a) rigid and b) flexible sandwiches and in each case their individual components

The rigid sandwich with core reinforcement does not have significantly better values than the unreinforced sandwich in the specific comparison (Fig. 7). The already very strong and stiff matrix is the reason for this. Increasing characteristic values with increasing material complexity can be detected in specific comparisons for the flexible materials.
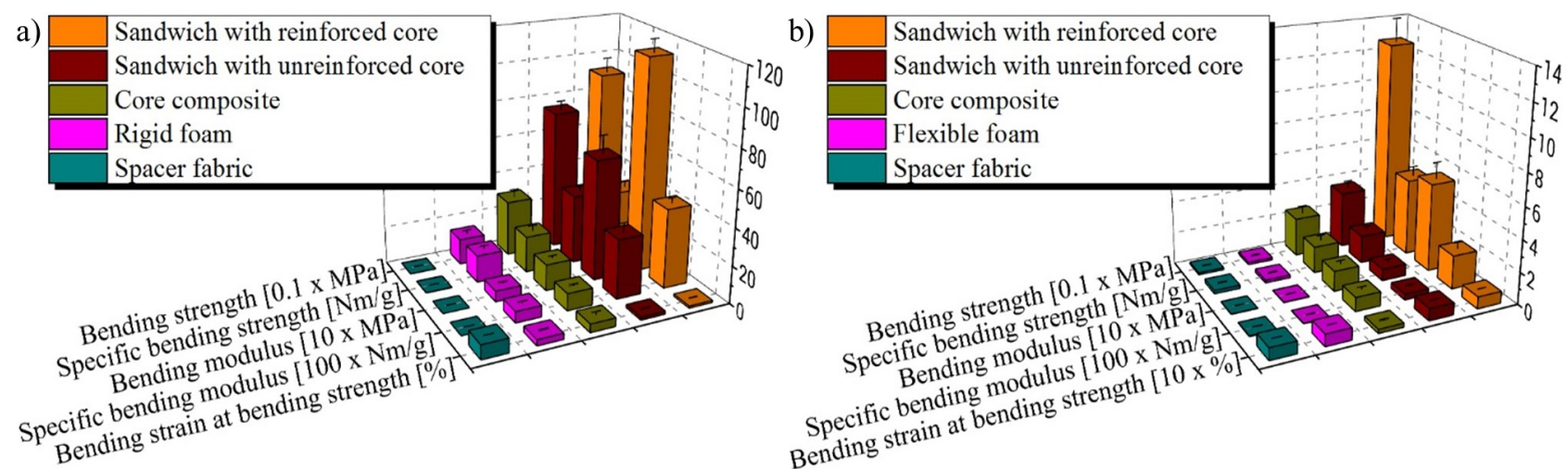

Fig. 7: Characteristic values of the bending test based on DIN EN ISO 178 of a) rigid and b) flexible sandwiches and in each case their individual components

\section{Summary}

This paper presents a developed sandwich consisting of thermoplastic composite facings and PUR foam cores with innovative spacer fabric reinforcement. The increase of the lightweight potential through the $3 \mathrm{D}$ endless fibre reinforcement in the core could be quantified on the basis of pressure and bending tests. This increases significantly in case of a flexible sandwich core. Only the absolute characteristic values can be increased for sandwiches with a rigid core. The additional mass resulting from the spacer fabric can only be compensated in the specific performance comparison. The reinforcement of the rigid foam core with spacer fabric makes it therefore unbreakable. 


\section{Acknowledgements}

This work was supported by the German Federal Ministry of Education and Research (Grant 03IPT508X) and the German Research Foundation (Grant EXC 1075).

\section{References}

[1] H.E. Friedrich, Leichtbau in der Fahrzeugtechnik, 2nd ed., Springer Vieweg, Wiesbaden, 2013.

[2] M. Vogt, Bestandsaufnahme Leichtbau in Deutschland, Projekt I C 4 - 10/15 Im Auftrag des Bundesministeriums für Wirtschaft und Energie, 2015.

[3] H. Nishida, V. Carvelli, T. Fujii, K. Okubo, Thermoplastic vs. thermoset epoxy carbon textile composites, IOP Conf. Ser.-Mat. Sci. Eng. 406 (2018) Article number 012043.

[4] J. Stiller, K. Schäfer, F. Helbig, J. Tröltzsch, D. Nestler, L. Kroll, Material selection and process configuration for free-form, voluminous and textile-based multi-material-design by the example of a bucket seat, Key Eng. Mat. 742 (2017) 302-309.

[5] P. Kasemphaibulsuk, M. Holzner, T. Kuboki, A. Hrymak, Foam injection molding of glass fiber reinforced polypropylene composites with laminate skins, Polym. Compos. 39 (2018) 4322-4332.

[6] G. Reyes, S. Rangaraj, Fracture properties of high performance carbon foam sandwich structures, Compos. Part A. 42 (2011) 1-7.

[7] M.R. Kamal, P. Singh, Q.M. Samak, S.M. Kakarala, Microstructure and Mechanical Behavior of Reinforced Reaction Injection Molded Polyurethane, Polym. Eng. Sci. 27 (1987) 1258-1264.

[8] F. Chen, C.P. Cao, W. Zhang, Y. Sun, Optimization of Parameters in Long Fiber Reinforced Reaction Injection Molding on Bending Properties, Adv.Mater. Res. 154-155 (2010) 981-986.

[9] S. Wirth, R. Gauvin, K.N. Kendall, Exp. Analysis of Core Crushing and Core Movement in RTM and SRIM Foam Cored Composite Parts, J. Reinf. Plast. Compos. 17 (1998) 964-988.

[10]X. Ye, H. Hu, X. Feng, Development of the Warp Knitted Spacer Fabrics for Cushion Applications. J. Ind. Textiles. 37 (2008) 213-223.

[11] S. Chen, H.-R. Long, Y.-H. Liu, F.-C. Hu, Mechanical Properties Of 3D-Structure Composites Based On Warp-Knitted Spacer Fabrics, Autex Res. J. 15 (2015) 127-137.

[12] K. Schäfer, B. Meier, S. Anders, F. Helbig, L. Kroll, Composites made from 3D warp-knitted textiles and polyurethane foam have a considerable reinforcing effect, KWP, (2014) 34-36.

[13] K. Schäfer, S. Valentin, B. Meier, I. Roth, F. Helbig, Comparing composites made from hard and soft materials: increasing the performance of rigid, hard pur foams by incorporating soft, elastic 3D warp-knitted textiles, KWP, (2014) 32-35.

[14] K. Schäfer, J. Stiller, J. Tröltzsch, D. Nestler, L. Kroll, Continuous, Free-Formable Sandwich Design with 3D Fiber Reinforced Core for Increased Lightweight Level of Applications in Large-Scale Production, Adv Eng. Mat. (2018) Article number 1800477.

[15] D. J. Nestler, Verbundwerkstoffe - Werkstoffverbunde: Status quo und Forschungsansätze, Habilitation, Universitätsverlag Chemnitz, ISBN 978-3-944640-12-9, 2014.

[16]K. Schäfer, S. Anders, S. Valentin, F. Helbig, J. Tröltzsch, I. Roth-Panke, D. Nestler, L. Kroll, Investigation of the specific adhesion between polyurethane foams and thermoplastics to suited material selection in lightweight structures, J Elastomers Plast. 50 (2018) 720-736.

[17]K. Schäfer, C. Göhler, J. Tröltzsch, D. Nestler, L. Kroll, Textile-based surface design of thermoplastic composites for microstructural adhesion to polyurethane foams for lightweight structures, submitted to Compos. Interfaces (2018).

[18] N. Brunk, E. Gründig, F. Helbig, M. Reinhardt, M. Scheika, C. Unger, Textile multiple-layer reinforcing structure having an integrated thermoplastic matrix for producing fibre-composite semifinished structures which can be shaped, WO patent 2012152242 A1 (2012). 